\title{
Article \\ Functional Symmetry after Surgical Treatment of Pertrochanteric Fractures in the Elderly
}

\author{
Karolina Gawronska ${ }^{1, *(\mathbb{D})}$ and Jacek Lorkowski ${ }^{2, *}$ \\ 1 Rehabilitation Centre, Central Clinical Hospital of the Ministry of the Internal Affairs and Administration, \\ 137 Woloska Street, 02-507 Warsaw, Poland \\ 2 Clinical Department of Orthopaedics, Traumatology and Sports, Central Clinical Hospital of the Ministry of \\ the Internal Affairs and Administration, 137 Woloska Street, 02-507 Warsaw, Poland \\ * Correspondence: kegawronska@gmail.com (K.G.); jacek.lorkowski@gmail.com (J.L.); \\ Tel.: +48-518958043 (K.G.); +48-606452887 (J.L.)
}

Citation: Gawronska, K.; Lorkowski, J. Functional Symmetry after Surgical Treatment of Pertrochanteric Fractures in the Elderly. Symmetry 2022, 14, 393. https://doi.org/ $10.3390 /$ sym 14020393

Academic Editor: Jan Awrejcewicz

Received: 6 January 2022

Accepted: 14 February 2022

Published: 16 February 2022

Publisher's Note: MDPI stays neutral with regard to jurisdictional claims in published maps and institutional affiliations.

Copyright: (C) 2022 by the authors. Licensee MDPI, Basel, Switzerland. This article is an open access article distributed under the terms and conditions of the Creative Commons Attribution (CC BY) license (https:/ / creativecommons.org/licenses/by/ $4.0 /)$.

\begin{abstract}
Pertrochanteric fractures (PFs) in the elderly and their consequences are among the leading causes of disability; they significantly reduce the quality of life and lead to loss of independence. This article aims to determine the functional and radiological outcomes in a group of patients with PFs treated with either the Dynamic hip screw (DHS) or intramedullary Gamma nail fixation. A total of 618 patients, admitted to hospital for pertrochanteric fractures between 2015 and 2019, at a mean age of 82.40 (range 29-104) were screened. Finally, 78 patients were enrolled. Parameters related to hospital stay and surgery (length of hospital stay, surgery duration) were compared in both groups. Functional outcomes were assessed by the Harris hip score, subjective pain was measured with a visual analogue scale (VAS), and quality of life was evaluated using the EQ-5D-5L questionnaire. The obtained results were evaluated at 3- and 6-month follow-up. Radiographic parameters were measured based on the preoperative and postoperative standing anterior-posterior pelvic radiographs and axial projection of the hip at 6-month follow-up. The results showed no significant difference between groups treated either with DHS or intramedullary Gamma nail fixation within the scope of the variables under study. In conclusion, both analysed methods support the functional symmetry of the musculoskeletal system.
\end{abstract}

Keywords: hip fractures; elderly; functional efficiency; quality of life; osteosynthesis; dynamic hip screw; intramedullary gamma nail

\section{Introduction}

Fractures of the proximal end of the femur, i.e., pertrochanteric fractures (PFs) and fractures of the neck of the femoral bone, are frequent lesions of the femur, which mainly affect elderly patients with osteoporosis and are typically caused by a low-energy trauma such as ground-level falls [1]. Statistically, one in three people in the world older than 65 years of age falls every year, and half fall at least twice [2,3]. It is estimated that $87 \%$ of fractures in the 65+ age group are caused by falls [4]. PFs occur more frequently in women than in men [5].

The occurrence of diseases especially those related to the cardiovascular system, nervous system, locomotor system, and involution changes associated with the aging process in some cases may lead to the so-called "death spiral" caused by injury.

The mortality rate remains very high, up to $30 \%$ in the first year after the injury [6]. Therefore, the prevention and treatment of these injuries still present major medical, social, and economic challenges for the healthcare system [2].

The diagnosis is most often made on the basis of interview, physical findings, and $X$-rays (pelvic radiograph in anterior-posterior (AP) projection and axial hip radiograph after injury). The use of computed tomography (CT) and magnetic resonance (MR) imaging 
is especially important for non-displaced fractures and for fractures found after a few weeks in a patient where the fracture healing process has already begun $[7,8]$.

The most commonly used for PFs are Boyd-Griffin, Evans, and nowadays, Arbeitsgemeinschaft für Osteosynthesefragen (AO) classifications, which classifies trochanteric fractures into three groups: A1-simple pertrochanteric fractures, A2-multifragmentary pertrochanteric, and A3-intertrochanteric $[9,10]$.

Surgery is the standard PF treatment [11]. It was proved that early treatment within $48 \mathrm{~h}$ after the injury, anatomical reduction of femoral fragments, and stable osteosynthesis are associated with better outcomes, reducing pain and allowing for early rehabilitation [11]. PFs are mainly surgically treated with the Dynamic hip screw (DHS), trochanteric gamma nails, and reconstruction nails [12,13].

The growing problem of an aging population and its consequences pose challenges to modern orthopaedics in the treatment of musculoskeletal injuries in people older than 65 years of age [14]. The skeletal biomechanical properties change with age, leading to osteoporotic fractures, in particular proximal femur fractures, including femoral neck and pertrochanteric fractures (both are life-threatening due to the immobilization of the elderly patient), distal forearm fractures, proximal humeral fractures, vertebral compression (mainly thoracic) fractures (not necessarily as a result of falls), and lower rib fractures [15]. According to Anadasivam et al., the most commonly injured bones are the tibia and fibula $(20.5 \%)$, ribs and sternum $(19.1 \%)$, and the proximal femur $(18.9 \%)$ [4]. Femoral neck fractures as well as pertrochanteric fractures (both types of injury in roughly equal numbers) account for $90 \%$ of all proximal femur fractures [1]. It is estimated that by 2050, the number of femoral fractures will increase from 6.3 to 8.2 million cases; therefore, the pertrochanteric fractures are going to be a real challenge to public health as the leading cause of orthopaedic hospitalization [16-18]. The standard treatment of PF fractures is surgery to allow a rapid mobilization of patients and prevent complications such as non-union and malunion of the proximal femur $[19,20]$.

The first documented use of external fixation to treat fractures dates back to 300-400 Before Christ. The first person to connect and immobilize bone fragments using a universal device was Hippocrates, who was also the first to describe a fracture fixation while preserving soft tissue integrity [21]. A. Lambotte proposed the term "osteosynthesis" in 1904, and the definition formulated by him in 1908, despite the passage of time, is still valid: "Osteo-synthesis is the artificial contention of the bone fragments of fractures, by special devices acting directly on bones, exposed or not, with the aim to strongly fix them in their original position" [22]. Depending on the degree of immobility at the fracture site under physiologic load, one can distinguish between absolute and relative fracture stability. The amount of stabilization determines the type of bone healing. Absolute stability is defined as the absence of motion between fracture fragments under functional load, obtained with a constant compression plates. Relative fracture stability is characteristic of external fixation of the fracture and allows small amounts of motion in proportion to the load applied [23].

The dynamic development of medical engineering based on artificial intelligence and nanotechnology has brought the need for intracorporeal stabilizers allowing the adequate stability to facilitate the fracture healing, reducing the number of potential complications and loss of function. The choice of a proper stabilization system remains under discussion and may vary among different medical centres [24]. Intramedullary nails are believed to have a biomechanical advantage, allowing stable fixation of more complex fractures without altering the anatomical conditions of the proximal femur, while preserving the lever arm of the abductor muscles. They can also be biologically advantageous as they can be implanted with a less extensive surgical approach, reducing blood loss and intraoperative soft tissue traumatization [25]. However, some authors say that implantation of the nail requires more advanced surgical skills because further fragmentation of bone fragments may occur during nail insertion. There is also a higher risk of embolism associated with the increase in pressure in the medullary cavity during reaming [26]. Based on a literature review, DHS fixation is used for stable fractures (according to AO from A1.1 to A2.1), while 
trochanteric nail fixation is used to treat unstable lesions (according to AO from A2.2 to A3.3). Proper fixation of the pertrochanteric fracture creates conditions for rapid recovery of locomotor functions of the patient after the surgery. Bone union disorders are rarely observed in this anatomical location because of the presence of richly vascularized "spongy" bone [27].

PFs in the elderly and their consequences are among the leading causes of disability; they significantly reduce the quality of life and lead to loss of independence and impaired social functioning. Therefore, PFs need multidisciplinary prevention and treatment [2]. Nowadays, in the era of revolution 4.0, in the treatment of pertrochanteric fractures, the principles of personalized medicine such as personalized implants and rehabilitation should be taken into account. This increases the possibility of getting good treatment results [28].

This study aims to determine the functional and radiological outcomes in a group of patients with pertrochanteric fractures treated with either DHS or intramedullary gamma nail fixation and particularly to:

- quantify and draw inferences on observed differences in parameters related to hospital stay and surgery (length of a hospital stay, surgery duration),

- quantify and draw inferences on observed differences in functional status at three and six months,

- quantify and draw inferences on observed differences in health-related quality of life (HRQoL) at three and six months between the study intervention groups,

- quantify and draw inferences on observed differences in the symmetry assessment in selected parameters based on the X-rays.

\section{Materials and Methods}

\subsection{Subjects}

A total of 618 patients (459 women, 159 men), admitted to hospital for pertrochanteric fractures between 2015 and 2019, at a mean age of 82.40 (range 29-104), participated in the study. The mean age of the hospitalized women was $83.77 \pm$ SD 9.94 (standard deviation), and the mean age of the hospitalized men was $78.43 \pm$ SD 14.49. A total of 314 cases of the right lower limb fracture and 304 cases of the left lower limb fracture were observed.

\subsubsection{Comorbidities}

The injuries associated with the trochanteric fracture included 11 fractures of the femoral bone, 20 of the leg bones, five fractures of the foot bones, 16 fractures in the humeral bone, 21 fractures of the forearm, and 15 in the wrist and hand bones. Among the admitted patients, there were 66 people with a past history of lower limb fractures (including 22 people who underwent hip arthroplasty and seven people who underwent knee replacement). A history of previous upper extremity trauma was noted in 37 people. Osteoarthritis was diagnosed in approximately 580 people admitted to the ward, with osteoporotic changes in 532 patients.

The most prevalent comorbidities were related to cardiovascular diseases: arterial hypertension (290 cases), ischemic heart disease (187 cases), including previous myocardial infarction (65 cases), heart failure (117 cases), atrial fibrillation (31 cases), heart rhythm disturbances (25 cases), mitral and tricuspid valve regurgitation (three cases), atrioventricular block (three cases), and hypertrophic cardiomyopathy (two cases).

Another group of comorbidities included diseases of the nervous system, such as a history of stroke/transient ischemic attack-TIA (46 cases), varying degrees of cognitive impairment (59 cases), Alzheimer's disease/dementia (32 cases), dizziness (30 cases), Parkinson's disease (15 cases), labyrinth disorders (six cases), psychomotor slowing (four cases), and epilepsy (three cases). Colon cancer was identified in 18 people, breast cancer in 14 people, pancreatic cancer in three people, stomach cancer in three people, bladder cancer in two people, melanoma in two people, and nose cancer in one person.

Among the diseases of the endocrine system, diabetes was reported in 62 hospitalized people (type 1: 19 people, type 2: 35 people, and in the remaining patients, the type was 
not specified in the history of the disease); nephropathy and diabetic retinopathy were found in a total of six people. Hypothyroidism and hyperthyroidism occurred in 28 and 14 patients, respectively. Respiratory diseases: asthma (11 cases), chronic obstructive pulmonary disease-COPD (39 cases), and emphysema (three cases).

Renal failure was also diagnosed in 23 people, nephrolithiasis in nine people, renal cyst in five, 2nd-degree hypertensive angiopathy in three people, hyperuricemia in three people, and urinary incontinence in 10 people. Further, 118 people were diagnosed with anemia and three with thrombocytopenia. Other diseases found in the patients included gastroenteritis (13 cases), gastritis (12 cases), polyps of the large intestine (nine cases), hyperlipidemia/dyslipidemia (nine cases), and diverticulosis of the colon (four cases). Biliary stones were reported in 19 patients, cirrhosis of the liver in eight patients, and acute viral hepatitis $C$ in seven. The following comorbidities were also found: leg ulceration (15 people), lymphoedema of the lower leg (10 people), venous insufficiency with postthrombotic syndrome in both legs (10 people), eye cataracts in nine people, and glaucoma in five people; 18 people were diagnosed with addictive disorders, including nicotine addiction in 12 people and alcohol dependence in six people. Some people were not fully diagnosed: upon hospital admission: 15 people presented with "generalized weakness," usually persisting for many months, who, after orthopedic treatment, were transferred to another hospital ward for further diagnosis and treatment.

\subsubsection{Procedures Performed}

All patients admitted to the hospital had preoperative A-P (anterior-posterior) X-rays of the pelvis upon the admission to the Accident \& Emergency Department, and 482 patients also had X-rays in axial projection. In the other $22 \%$ of cases, the X-rays were performed in the indirect projection between the A-P and axial projection due to lack of cooperation with the patient or pain sustained while performing the X-ray.

Due to the accompanying injuries and comorbidities of the motor organ, $\mathrm{X}$-ray images of the thigh/knee/shank were taken in 84 patients and X-ray images of the ankle/foot $\mathrm{X}$-ray in nine patients. X-ray images of the shoulder and arm were performed in 20 people, $\mathrm{X}$-rays of the elbow/forearm bones in 24 people, and X-rays of the wrist bones in 17 people.

All hospitalized patients underwent anaesthesia consultation before the surgery, with additional tests performed beforehand, and medical consultations whenever deemed necessary.

Additionally, the following preoperative consultations were carried out: general practitioner (280 patients), cardiologist (164 patients), including echocardiogram (73 patients) and cardiac stimulation (14 patients), diabetologist (92 patients), gastrologist (63 patients), including ultrasonography of the abdomen and retroperitoneal space (31 patients), neurologist (47 patients), general surgeon (39 patients), vascular surgeon (23 patients), including peripheral vascular ultrasound (10 patients), nephrologist (21 patients), oncologist (15 patients), pulmonologist (12 patients), laryngologist (three patients), and ophthalmologist (three patients). Some of these consultations were repeated or carried out in the postoperative period. Before the operation, all patients had an electrocardiogram and a chest X-ray.

Before the surgery, all patients were examined for the following parameters: blood group, serological compatibility test, and blood count, sodium, potassium, creatinine, activated partial thromboplastin time (APTT), and prothrombin time (PT). A total of 59 patients needed supplementation to increase red blood cell counts before the surgery, and 26 patients needed supplementation of plasma.

Repeated X-rays in the AP and axial projection were taken one day after surgery in all patients. On the day of the surgery or on the following days, 446 people received 1-2 units of red blood cell concentrate in the postoperative period, 39 people received three units of red blood cell concentrate, and three people received four or more units of the above-mentioned concentrate. Laboratory tests were performed in the late evening hours or in the morning for 40 people operated in the afternoon or in the evening, who were 
administered a red blood cell concentrate immediately after or during the surgery; plasma was transfused 160 times.

A complete blood count, sodium, potassium, creatinine, activated partial thromboplastin time (APTT), and prothrombin time (PT) were repeated in the evening hours on the day of surgery, as well as on the 1st, 2nd, and 3rd day after surgery in the early morning hours. The same tests, according to the standard of the hospital, were repeated in approx. $14 \%$ of patients, i.e., those with prolonged hospitalization due to general and local complications, including exacerbation of chronic diseases and postoperative wound healing disorders.

During the stay in the orthopaedic ward, other laboratory tests and procedures, such as a CT of the head without contrast in 28 patients, C-reactive protein- 866 times, total protein-80, were also performed. The remaining laboratory tests were conducted 1470 times.

During the stay in the ward, subjects underwent a standard post-operative rehabilitative protocol (when allowed by the patient's clinical status and osteosynthesis stability) and were diagnosed within the scope of physiotherapy in accordance with the hospital standards. The rehabilitation program was intended to minimize the risk of complications after pertrochanteric fractures and counteract the effects of long-term immobilization. The first day after surgery included isometric exercise of quadriceps and gluteal muscles, anticoagulant exercises, and respiratory exercises. On the second day after surgery, the following exercises were added: active slow exercises of lower extremities, gait re-education (passive verticalization, active verticalization, and assisted walking with weight-bearing tolerance using a Zimmer frame). In the absence of cooperation with the patient, the program provided for rehabilitation with a physiotherapist-passive mobilization.

Repeated X-ray images, (X-ray of the pelvis with the hip joints and axial X-ray of the operated hip) taken as part of post-hospital/outpatient treatment in patients who attended follow-up visits in the orthopaedic clinic approximately six weeks after the operation, were performed in 502 patients. Approximately three months after the surgery, $\mathrm{X}$-rays were taken in 389 people, and six months after the surgery-in 308 people. In the post-hospital period, home physiotherapy was continued in accordance with the plan of physiotherapeutic treatment in patients after fixation of a pertrochanteric fracture.

\subsubsection{Types of Surgical Fixation}

Out of 618 patients admitted to the ward with a diagnosis of a pertrochanteric fracture, 597 patients underwent fracture surgery, including 441 fixations with a DHS screw-plate (Figure 1A,B), 141 using an intramedullary Gamma nail (Figure 2A,B), and the remaining 15 patients were treated with ZESPOL (proper name) stabilizers-five patients, three cannula screws - one patient, and bipolar prostheses-nine patients. The DHS screws used included 3-hole plate-screws (305 pieces) and 4-hole plate-screws (136 pieces).

An anti-rotation pin was used on 36 intramedullary Gamma nails. The pin was used to prevent rotation during surgery when inserting the chuck screw and in the postoperative period, especially during kinesiotherapy. Some patients ( 21 people) were disqualified from the fixation surgery by the decision of an anaesthesiologist.

In the case of fixation with the DHS screw-plate, the size of the skin incision, allowing surgical access to the operating field, was $10-12 \mathrm{~cm}$ and about $16 \mathrm{~cm}$ in 31 cases.

In the case of fixation with a Gamma nail, three standard incisions for inserting the nail, neck screw, and 1-2 lower locking screws were $2-4 \mathrm{~cm}$ long. In 11 cases, it was necessary to connect two upper cuts for optimal fracture reposition.

In the case of fixation with DHS, drainage was performed on 338 people, whereas in the case of intramedullary Gamma nail, drainage was performed on nine people. The drain was removed on the first or the second day after the surgery. The surgical dressing was changed every $1-2$ days. 


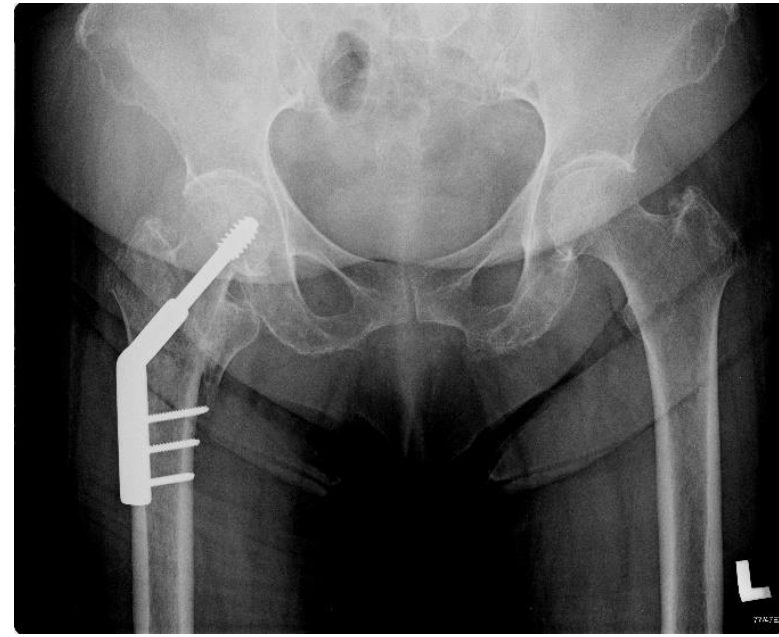

(A)

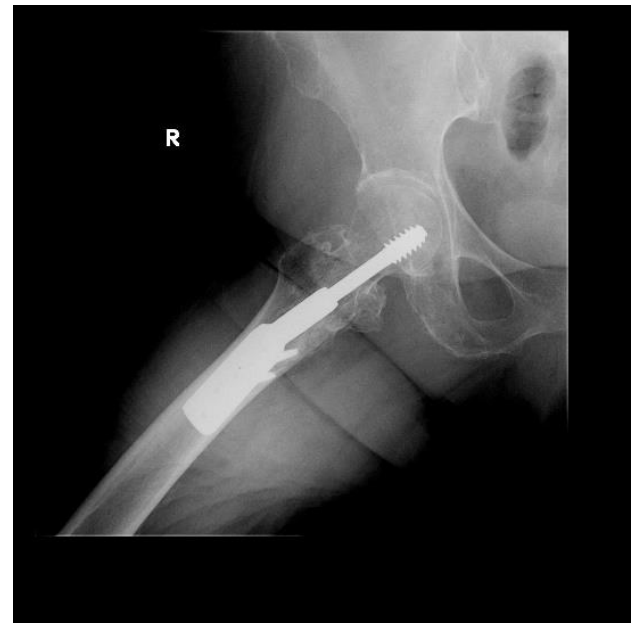

(B)

Figure 1. X-ray image after stabilization of the pertrochanteric fracture with the DHS methodcomplete fracture healing anterior-posterior (A) and axial X-ray view (B).

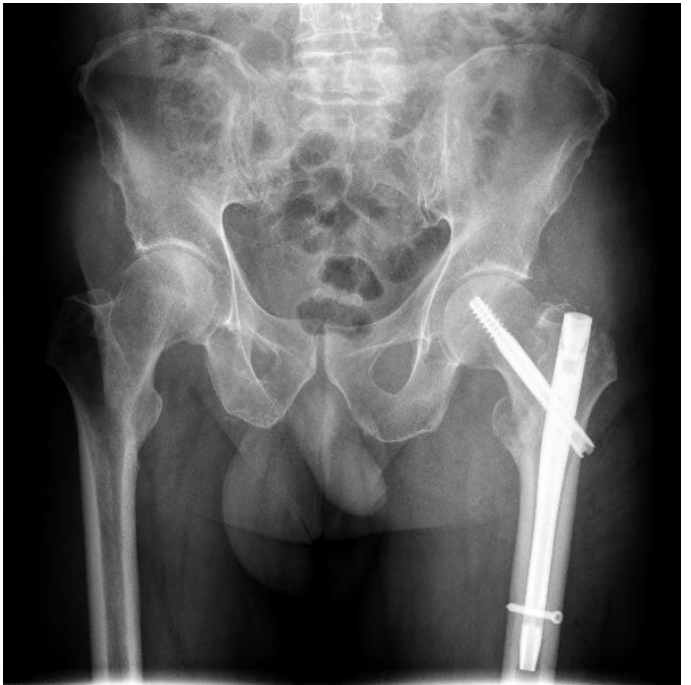

(A)

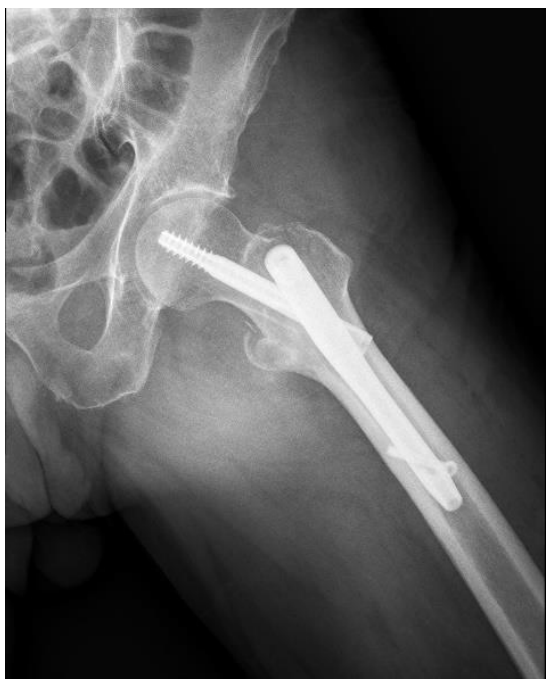

(B)

Figure 2. X-ray image after stabilization of the pertrochanteric fracture with a gamma nail—complete fracture healing anterior-posterior (A) and axial X-ray view (B).

\subsubsection{Study Group}

For more detailed analysis, patients who underwent surgical fixation of pertrochanteric fracture by a single experienced surgeon were enrolled in the study.

\subsection{Inclusion Criteria}

- Patients admitted for a hospital stay in the department of orthopaedics and traumatology of the motor organ in the period from 1 January 2015 to 31 December 2019 with the primary diagnosis of pertrochanteric fracture;

- patients who underwent fixation of pertrochanteric fracture, performed by one/the same operator (operating surgeon-consultant);

- type of fixation used: DHS screw plate or intramedullary Gamma nail;

- type of fracture according to AO classification-A1, A2, A3;

- $\quad$ aged $65+$;

- a minimum post-operative follow-up of six months. 


\subsection{Exclusion Criteria}

- $\quad$ Patients who had a poor outcome due to comorbidities, who did not report to outpatient control;

- $\quad$ a different type of fracture fixation was used;

- multi-site musculoskeletal injuries;

- pathological fracture (excluding osteoporosis).

The final number of subjects enrolled in our study was 78 (36 in the DHS group and 42 in the GAMMA group). Between the DHS and GAMMA groups, no statistical differences in the sex and age distribution emerged. The study was approved by the Ethical Board of our hospital.

\subsection{Symmetry in the Length of Hospital Stay and Surgery Duration}

The subjects staying in hospital were compared using the following parameters:

1. The length of hospital stay following the day of surgery admission (including the day of surgery)-days;

2. The duration of the hospital stay after surgery (days);

3. The duration of surgery (from anasthesia, including positioning and repositioning of the patient of the operating table) - min.

\subsection{Functional Symmetry}

Clinical data were obtained from multiple sources: reviewed retrospectively from the patients' charts, other hospitals' discharge summaries, and during follow-up visit as part of an outpatient treatment three and six months after the surgery. Clinical outcome was assessed using the Harris hip score (HHS) [29]. The HHS scale was developed for the assessment of the results of hip surgery and is intended to evaluate various hip disabilities and methods of treatment in an adult population, including functional outcome after pertrochanteric hip fracture [30-32]. The scale applied in the study consists of 10 items covering domains of pain, function, functional activities, deformity, and hip range of motion. The interpretation of outcome using the modified Harris hip score was as follows: $<70$ (poor result), 70-79 (fair result), 80-89 (good result), and >90 (excellent result). Additionally, we noted subjective pain by a visual analogue scale (VAS, range from 0 to 10).

The EQ-5D-5L questionnaire was used to measure the health-related quality of life [33]. The EQ-5D-5L is a validated, standardized, and responsive instrument that comprises a visual analogue scale recording self-rated health and current health status, consisting of five response levels (no problems, slight problems, moderate problems, severe problems, and extreme problems) for five dimensions: (1) mobility, (2) self-care, (3) usual activities, (4) pain/discomfort, and (5) anxiety/depression. The EuroQol visual analogue scale scores self-rated health on a scale on which the end points are labelled 'best imaginable health

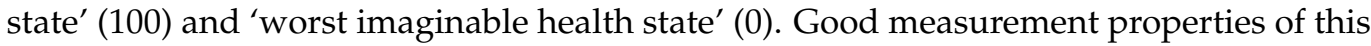
tool have been confirmed by other researchers in a similar cohort of patients with fragility hip fracture [34,35].

\subsection{Radiographic Analysis}

Radiographic parameters were measured based on the preoperative and postoperative standing AP pelvic radiograph and axial projection of the hip at 6-month follow-up. The literature was taken into account when selecting the radiological parameters to be assessed [36-38].

Radiographs were assessed for (Figure 3):

- $\quad$ fracture union (full union vs. non-union present);

- $\quad$ symmetry of the neck-shaft angle (NSA)-operated femur and its opposite side (assessment for the presence of valgus or varus position);

- $\quad$ symmetry of the position of the neck screw in relation to the axis of the femoral neck (screw located on the axis or below the axis vs. located above the axis); 
- the axis of the intramedullary nail in relation to the axis of the femur (evaluated in the anterior-posterior view: varus position-the distal part of the nail resting on the medial cortex; axial position-the axis of the nail coincides with the axis of the femoral shaft; valgus position-the distal part of the nail resting on the cortex layer of the femur on the lateral side);

- the minimum distance between the tip of the neck screw and the articular surface of the femoral head (arithmetic mean of the distance measured in the anterior-posterior and axial projection);

- $\quad$ the fracture/detachment of the lesser trochanter (X-ray of the hip after injury, before surgery);

- $\quad$ symmetry of the length of the operated limb (the difference in the height of the lesser trochanter position) shortening by $>1 \mathrm{~cm}$.

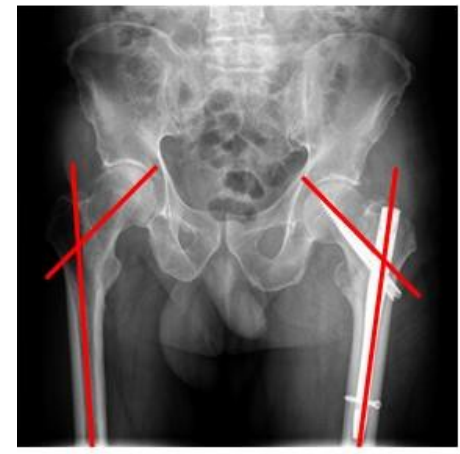

A

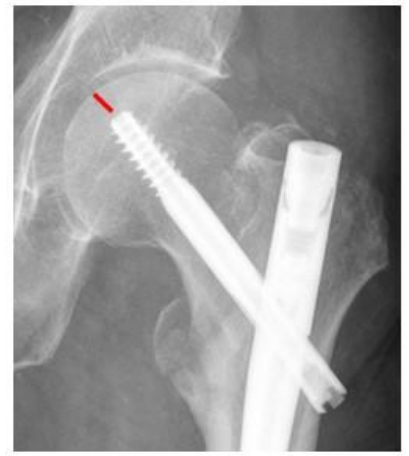

D

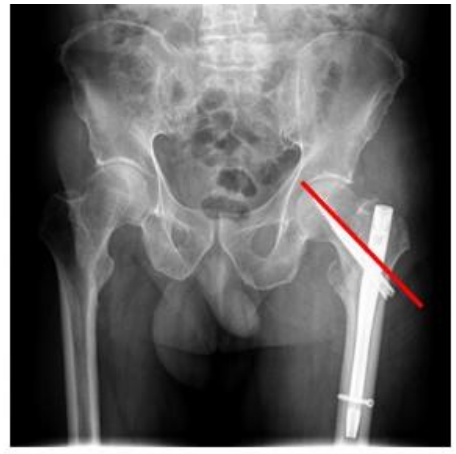

B

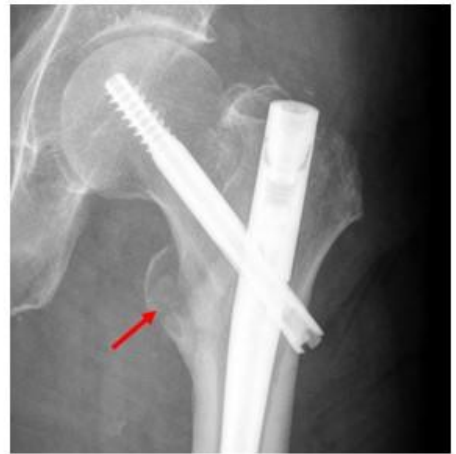

E

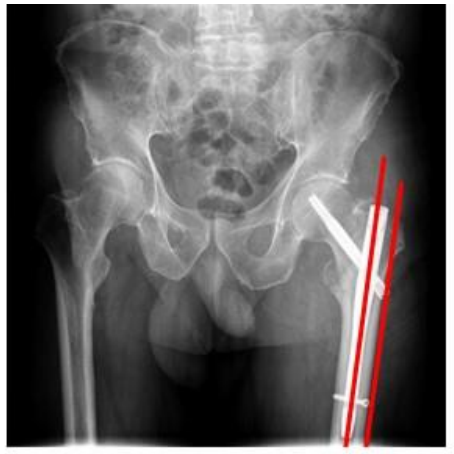

C

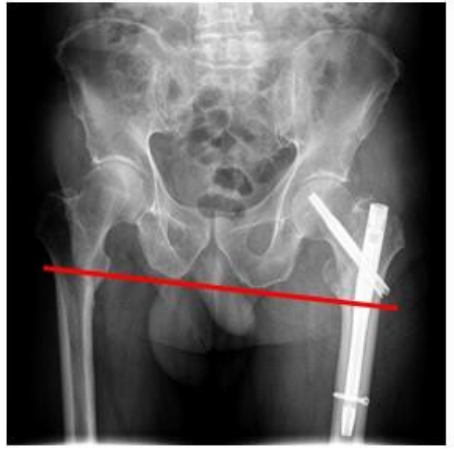

$\mathbf{F}$

Figure 3. Assessment method: (A) neck-shaft angle (NSA)_operated femur and its opposite side (assessment for the presence of valgus or varus position); (B) the position of the neck screw in relation to the axis of the femoral neck (screw located on the axis or below the axis vs. located above the axis); (C) the axis of the intramedullary nail in relation to the axis of the femur (evaluated in the anterior-posterior view: varus position-the distal part of the nail resting on the medial cortex; axial position - the axis of the nail coincides with the axis of the femoral shaft; valgus position - the distal part of the nail resting on the cortex layer of the femur on the lateral side); (D) the minimum distance between the tip of the neck screw and the articular surface of the femoral head (arithmetic mean of the distance measured in the anterior-posterior and axial projection). The figure shows the determination of distances in the anterior-posterior projection of the X-ray. This distance is determined analogously in the axial projection; (E) the fracture/detachment of the lesser trochanter (X-ray of the hip after injury, before surgery); (F) shortened length of the operated limb (the difference in the height of the lesser trochanter position) by $>1 \mathrm{~cm}$.

\subsection{Statistical Analysis}

Statistical analyses were performed with Statistical software (Statistica 10.0). The data were normally distributed, and independent-sample $t$-tests were used. The level of statistical significance was set at $p<0.05$ for all analyses. 


\section{Results}

\subsection{Symmetry in the Length of Hospital Stay and Surgery Duration}

The DHS group consisted of 36 subjects, and the Gamma group included 42 subjects. Types of fractures according to AO classification:

- In the DHS group: (31A1-eight cases, 31A2-28 cases);

- In the Gamma group: (31A1—five cases, 31A2-30 cases, 31A3—seven cases).

Regarding side fracture, in the DHS group: 17 on the left side and 19 on the right side. In the Gamma group: 19 on the left side and 23 on the right side.

As shown in Table 1, no statistical difference was reported with regard to the length of hospital stay (time from admission to surgery and from surgery to discharge-days) and intra-operative time (in $\mathrm{min}$ ).

Table 1. Comparison of parameters related to hospital stay and surgery in the DHS group $(n=36)$ and Gamma group $(n=42)$.

\begin{tabular}{cccc}
\hline & DHS Group & Gamma Group & $p$ \\
\hline Time from admission to surgery (days) & $3.18 \pm 1.85$ & $3.38 \pm 1.70$ & 0.57 \\
Time from surgery to discharge (days) & $4.96 \pm 2.86$ & $4.31 \pm 2.22$ & 0.21 \\
Intra-operative time (min) & $52.56 \pm 15.32$ & $54.33 \pm 19.48$ & 0.62 \\
\hline
\end{tabular}

Values are expressed as the mean \pm standard deviation.

\subsection{Symmetry in Functional Outcomes}

Regarding functional outcomes assessed using the Harris hip score, there was no difference between the two groups at 3-month and 6-month follow-up (Table 2). Functional symmetry was maintained between the two study groups.

Table 2. Comparison of outcomes using the Harris hip score in the DHS group $(n=36)$ and Gamma group $(n=42)$ three and six months after surgery.

\begin{tabular}{cccc}
\hline & DHS Group & Gamma Group & $p$ \\
\hline HHS 3-month follow-up & $67.43 \pm 7.07$ & $68.33 \pm 6.01$ & 0.54 \\
HHS 6-month follow-up & $78.36 \pm 5.52$ & $79.03 \pm 5.18$ & 0.58
\end{tabular}

Values are expressed as the mean \pm standard deviation.

As shown in Table 3, no statistical difference in subjective pain assessed with the visual analogue scale (VAS) was reported at three and six months post-operatively, and functional symmetry was maintained.

Table 3. Comparison of outcomes of subjective pain by VAS in the DHS group $(n=36)$ and Gamma group $(n=42)$ three and six months after surgery.

\begin{tabular}{cccc}
\hline & DHS Group & Gamma Group & $p$ \\
\hline VAS (pain) 3-month follow-up & $3.72 \pm 1.03$ & $3.66 \pm 0.93$ & 0.80 \\
VAS (pain) 6-month follow-up & $2.44 \pm 0.94$ & $2.21 \pm 0.98$ & 0.29 \\
\hline
\end{tabular}

\subsection{Symmetry in Quality of Life}

Comparing the quality of life of both groups, as shown in Table 4, data collected using the EQ-5D-5L questionnaire, including the EuroQol Visual Analog Scale (VAS) measuring scores of self-rated health, showed no statistical difference. Functional symmetry between the study groups in terms of quality of life was maintained. 
Table 4. Comparison of parameters related to quality of life in the DHS group $(n=45)$ and Gamma group $(n=52)$ assessed by EQ-5D-5L three and six months after surgery. The first two results are shown as a 'value sets' - a single summary index value of the health status.

\begin{tabular}{cccc}
\hline & DHS Group & Gamma Group & $p$ \\
\hline 3 months post-operatively & $0.59 \pm 0.12$ & $0.60 \pm 0.11$ & 0.73 \\
6 months post-operatively & $0.64 \pm 0.09$ & $0.63 \pm 0.09$ & 0.58 \\
VAS 3 months post-operatively & $55.55 \pm 7.45$ & $56.69 \pm 5.84$ & 0.45 \\
VAS 6 months post-operatively & $61.97 \pm 9.24$ & $62.61 \pm 7.60$ & 0.73 \\
\hline
\end{tabular}

Values are expressed as the mean \pm standard deviation.

The first two results are shown as a 'value sets' - a single summary index value of the health status. A value set provides values (weights) for each health state description according to the preferences of the general population of a region.

\subsection{Symmetry/Assymetry in Radiographic Analysis}

On the basis of X-ray images, bone union was found in 78 cases.

Table 5 shows the comparison of the radiological parameters between the DHS and Gamma groups. The mean values of the minimum distance between the tip of the neck screw and the articular surface of the femoral head were greater in the DHS group than in Gamma group (14.96 vs. 10.33). This tested parameter indicates the asymmetry between the studied groups. In the other tested parameters, the obtained results did not indicate any significant difference in the distribution between both groups.

Table 5. Comparison of parameters related to selected parameters based on the X-rays in the DHS group $(n=36)$ and Gamma group $(n=42)$.

\begin{tabular}{cccc}
\hline & DHS Group & Gamma Group & $p$ \\
\hline NSA-non-operated side (degrees) & $131.61 \pm 2.47$ & $130.76 \pm 1.90$ & 0.09 \\
NSA-operated side (degrees) & $133.22 \pm 3.07$ & $132.07 \pm 2.87$ & 0.09 \\
$\begin{array}{c}\text { NSA difference between the operated and } \\
\text { the opposite side (degrees) }\end{array}$ & $2.36 \pm 2.11$ & $2.17 \pm 2.18$ & 0.69 \\
$\begin{array}{c}\text { The minimum distance between the tip of } \\
\text { the neck screw and the articular surface of } \\
\text { the femoral head (millimeters) }\end{array}$ & $14.96 \pm 5.39$ & $10.33 \pm 3.40$ & $0.00 *$ \\
$\begin{array}{c}\text { Values are expressed as the mean } \pm \text { standard deviation. }{ }^{*} p<0.05 . \\
\end{array}$ & & \\
\hline
\end{tabular}

The position of the neck screw in relation to the axis of the femoral neck:

1. DHS group: in 33 patients, the screw was located on the axis or below; in three patients, the screw was located above the axis,

2. Gamma group: in 38 patients, the screw was located on the axis or below; in four patients, the screw was located above the axis.

The axis of the intramedullary nail in relation to the axis of the femur: varus positionnine subjects; axial position-30 subjects; valgus position-three subjects.

The fracture/detachment of the lesser trochanter in the DHS group-23 subjects, Gamma group-27 subjects.

The difference in the height of the trochanter position is the smaller/shortened length of the operated limb by $>1 \mathrm{~cm}$ : in the DHS group—three subjects, Gamma group: five subjects.

\section{Discussion}

Bipedality is one of the hallmarks of a human being [39]. The dominance of the right upper limb in 90 percent of the population is a well-known fact [40]. This must lead to laterality. Lateralization is defined as the different specialization of the function of the left and right sides. It begins at the level of the central nervous system. Lateralization is a common phenomenon in humans, mammals, and throughout the animal kingdom [41]. 
This phenomenon should lead to asymmetry in organ function. This applies in particular to the musculoskeletal system, which, in its anatomical structure, has symmetrical structures. Their function is determined by the nervous system. Simply put, the nerve pathways, in particular the pyramidal pathways, that is, the cortico-nuclear and cortico-spinal pathways, are responsible for arbitrary movements, and the extrapyramidal paths are responsible for involuntary movements. Complex synaptic processes within the central nervous system are required before this stage occurs. They are based on the stimuli provided by the ascending nerve pathways. These are the sensual end sensory nerve pathways. Their anatomical structure is symmetrical, but not fully symmetrically functional [42]. The asymmetrical use of limbs to handle objects or perform motor activities is widely known [43]. Similarly, there is asymmetrical use of sensory paired organs, such as eyes and ears in order to perceive the received stimulus $[44,45]$. Lateralization differs in strength (a person may be more or less lateralized) [46]. Having an asymmetric brain has several advantages [46-48]. Saving energy is one of them. Entropy is a measure of the degree of disorder in a system and energy dissipation. It is extensive. According to the second law of thermodynamics, if a thermodynamic system moves from one state of equilibrium to another without external factors (i.e., spontaneously), its entropy always increases [49]. This is essential for cognitive tasks. It avoids duplication of functions. Another significant gain resulting from lateralization is the problem of preferential use of the left and right hemispheres of the brain in specific tasks [50,51].

The question arises whether the asymmetry in the functions of the cerebral hemispheres and the dominance of one of the limbs cause differences in the manner of collapse? Our observations on the 78 groups of people of senior age did not indicate this. A pertrochanteric fracture occurred in 42 subjects on the right side and in 36 subjects on the left side. There were no statistically significant differences. Other researchers reported similar results [36]. This indicates a balance between almost complete anatomical symmetry and functional dominance of one of the limbs.

It is interesting to restore the functional balance almost completely after trauma and surgery. This observation is made indirectly. Our results on the neck-shaft angle and the length of the operated limb showed the reconstruction of the symmetry of the musculoskeletal system in terms of anatomy. The differences in its scope are slight. A good functional result (Harris hip score) indicates that an asymmetry to disable the function is not possible. The existing asymmetry may be treated as small enough to be treated by the patient as physiological. Therefore, it can be assumed that the symmetry is maintained after the treatment of pertrochanteric fractures. Other authors also do not see any disturbances in this symmetry [13].

Lateralization has been described not only in humans but also in many species of animals, not only mammals. Interesting observations are found in cuttlefish: left-turning biases in cuttlefish result from an eye use preference. Emergence of visual lateralization at the time of dispersal may afford juveniles greater behavioral efficiency by enabling them to look out for escape routes while hunting [52]. The results of similar studies would be interesting in humans. As a result of injuries, a high coefficient of maintaining symmetry was found in the group we studied. This seems inconsistent with the observations in animals in the case of the natural course of physiological processes. New interesting solutions in research can bring ethnorobotics. This is a new fascinating feld of biorobotics, proposing the use of robotic replicas as an advanced method for investigating animal behavior. This novel research approach can also encourage the development of advanced bioinspired robots [53]. Robotics can be used not only to study animals but also to treat humans [54]. However, its use has not yet been described for the treatment of pertrochanteric fractures.

Lateralization is a fundamental principle that may have evolved from a common ancestor to vertebrates and invertebrates or it may have separately evolved in a convergent evolutionary proces $[55,56]$. This is true. However, in the case of surgical intervention aimed at recreating the anatomy of its deepening in an excessive way, it has not been found. 
The stated one seems to be more related to the degree of destruction of the locomotor system as a result of the trauma. This is what our research shows.

The present study revealed no significant difference in intra-operative time and hospital stay between patients treated with DHS and those treated with the intramedullary Gamma nail. A prospective randomised trial conducted by Guyer et al. showed that the operation time in the Gamma nailing group was statistically longer than that in the DHS group [57]. Selim et al. reported a longer intra-operative time (defined as skin-to-skin) in the DHS group-116 $\pm 32.49 \mathrm{~min}$ (mean, SD). However, this study group also had a Trochanteric stabilizing plate (TSP), as a modular extension of the DHS, used to stabilize the greater trochanter and lateral wall [36].

As reported by Catania et al., the mean operative time in the Gamma group was $42 \pm 7.4 \mathrm{~min}$ (mean, SD) [13]. Our findings revealed a longer intra-operative time. This could be due to a different approach applied to calculate the intra-operative time, i.e., from anaesthesia, including positioning and repositioning of the patient on the operating table.

Early treatment is one of the factors determining better outcomes [13]. Hospital stay duration from admission to surgery and from surgery to discharge was similar in both groups. The median length of a hospital stay shorter than that reported in our study-two days on average from admission to surgery-was reported by Griffin et al. [38]. This could be due to our calculation method that included the day on which the operation was performed. The length of hospital stay (from admission to discharge) in the DHS group was similar to the results obtained by other researchers (mean of eight, 15-day hospital stay) [36].

Based on the literature review, hip fractures have an adverse effect on the quality of life of people who sustained fractures, both in the pre- and postoperative period, in short and long-term observations. Gjersten et al. presented long-term results claiming that fracture of the femur significantly reduced the quality of life of the patients. The study included 10,324 patients who, six months after sustaining the fracture, did not return to the pre-injury state [58]. Peeters et al. drew attention to the fact that the healthrelated quality of life most considerably improved during the first six months after hip fracture in the elderly [59]. Based on the demographic characteristics of the test group, we found no significant differences between the two groups in terms of a subjective assessment of health-related quality of life. These data could be due to the fact that the operations were performed by the same experienced surgeon and by using the standard rehabilitation protocol for subjects operated on with DHS and the intramedullary Gamma nail. No significant difference in the assessment of the quality of life was reported by Griffin et al.; however, the study conducted by his team concerned people operated on with an intramedullary nail and fixed-angle plate fixation [38]. EQ VAS provides a quantitative measure of patients' perception of their health, which accounted for $50.4 \pm 20.9$ (mean, SD) in patients at four months after surgery with an intramedullary nail, while in our study, it amounted to $55.55 \pm 7.45$ (mean, SD) at 3-month follow-up.

With regard to functional outcomes measured using the HHS questionnaire, there was no significant difference observed between both groups at 3- and 6-month followup. The lack of a significant difference between the operated groups was also confirmed by Chang et al. [60]. These results may be due to the same physiotherapy treatment used and operations performed by the same surgeon. Selim et al. conducted a study with 20 patients who underwent DHS + TSP fixation. A mean HHS score at 3-month follow-up was $72.53 \pm 12.42$ (mean, SD), and at 6-month follow-up, the score was $83.32 \pm 9.73$ (mean, SD) [36]. The functional outcome using HHS in the Gamma nail group at 6-month follow-up, according to Catania et al., was better than that reported in our study (84.25 versus 79.03) [13]. Regarding the perceived pain assessed with the VAS method, the two groups did not demonstrate differences at 3- and 6-month follow-up. The results may be due to the treatment provided by the same orthopaedist and with a standardized rehabilitation protocol. 
We observed fracture healing in the radiological image in each case analysed in the study group. In the literature, non-union/malunion was not found even in a few percent of patients [61]. However, our results are not fully comparable in this case due to the exclusions in the research methodology. Considerable literature has developed on the assessment of the radiological examination results performed in patients with pertrochanteric fractures; however, the presented methods of evaluating the treatment effects are not always consistent with each other [36-38].

In our opinion, the most important result obtained in our group is the symmetry between the operated and the non-operated side. This happened despite the necessity to recreate the anatomy of the operated side of the osteoporotically affected limb. This applied to fractures treated with both a Gamma nail and the DHS method. Hence, it seems that the division of the neck-shaft angle into normal, valgus, and varus, presented most often in the literature, is insufficient $[37,38]$. Based on X-rays, when full union was achieved in the entire study group, these values were within the normal range, but the physiological values for individual patients were different. Therefore, comparing the results of studies taking into account only operated limbs across different populations may be biased. Thus, we found it important to compare the results of the operated limb with the opposite side. We obtained statistically significant symmetry, and this seems to be a good result.

The position of the neck screw in relation to the axis of the femoral neck is another parameter subject for analysis [37]. The screw, in accordance with the applicable standards, in the majority of cases was located on the axis or below the axis of the femoral neck (in 69 subjects). A careful analysis of cases where the screw was placed above the axis points out that this was due to the primary microanatomy changes in the neck of the femoral bone. The destruction of trabecular bone tissue in this location is the most common change of this type, making it impossible to implant the screw in the correct position while maintaining the appropriate biomechanical efficiency. Thus, it is important that the alternative surgical approach proves to be effective. In the case of intramedullary nails, their axial insertion into the femur was additionally assessed. This was achieved in 30 cases. Due to the multifracture destruction of the trochanteric region, which partially determines the stabilization method, it seems to be a good result. The most precise studies described in the literature were carried out in this case with regard to changes in the re/procurvatum position, but those in the varus/valgus scope are significant [62].

The minimum distance between the tip of the neck screw and the articular surface of the femoral head was another parameter that we examined. It was checked in terms of stabilization with both the Gamma nail and DHS method. Most authors, according to the literature, evaluate this case with TAD (tip-apex distance) [5]. We are not assessing the TAD at this point; it will be the subject of our further research. It has great value. The method proposed in this study is simpler and allows for measurement of another important parameter, namely, the risk of perforation of the cortical layer and penetration of the screw into the joint. This is a significant complication that was not present in the study group. This complication cannot be assessed and described by TAD.

We found fracture and dislocation of the lesser trochanter in 50 cases.

Fractures of the lesser trochanter according to the AO classification are found in a significant part of A1- and A2-type fractures [11]. In each analysis of the biomechanics of the hip joint and fractures in this area, the iliopsoas muscle and its attachment to the lesser trochanter should be taken into account.

Shortening of the operated limb is an essential element of treatment from the biomechanical and clinical point of view. In four cases, it was needed due to the difference in the degree of NSA varus in the operated and non-operated limbs, despite the fact that the NSA was within the normal range, and in four cases due to compression at the fracture site. We took into account the commonly used limit of 1 centimetre in our ratings. Among the patients suffering such a distortion were those operated with both the DHS method and the Gamma nail (3 vs. 5). The distortion was more prevalent in the case of the Gamma nail, 
even though it should be remembered that the analysed group included only 78 people, and that the Gamma nail was used to stabilize more unstable, multi-fragmented fractures.

In this article, we did not take into account other, in our opinion, valuable parameters, but we selected those that seemed most meaningful. The analysis of the effects of X-ray data obtained indicate a good treatment outcome, comparing the stabilization of the fracture with the Gamma nail and the DHS method. Gamma nails, however, seem to be better, considering the fact that they stabilize fractures that are more difficult to stabilize.

There may be some possible limitations in this study. The first one concerns the limited number of enrolled patients. Findings presented herein were observed in a cohort of only 78 individuals. Moreover, the short follow-up period does not allow for a long-term evaluation of the results. Extending the follow-up period to one year could show the difference between the study groups, e.g., functional asymmetry. The lack of comparison of complications after surgery is another potential limitation. Finally, the degree of preexisting comorbidities and biomechanical analysis were not considered; these factors may have affected the quality of the measurement.

\section{Conclusions}

1. There was a functional symmetry between the outcomes of surgical treatment of PFs in people treated with the dynamic hip screw and intramedullary gamma nail.

2. There were no significant asymmetries in parameters regarding hospital stay and surgery between the DHS group and Gamma group.

3. There were no significant asymmetries in parameters regarding functional status (motor function examined by assessing the performance of specific motor tasks), perceived pain, and quality of life at three and six months after surgery in both groups.

4. There were no significant asymmetries in the radiographic image of the hip after the surgery of the pertrochanteric fracture and the hip on the opposite side.

Author Contributions: Conceptualization, J.L. and K.G.; methodology, J.L. and K.G.; software, J.L. and K.G.; validation, J.L. and K.G.; formal analysis, J.L. and K.G.; investigation, J.L. and K.G.; resources, J.L. and K.G.; data curation, J.L. and K.G.; writing-original draft preparation, J.L. and K.G.; writing-review and editing, J.L. and K.G.; visualization, J.L. and K.G.; supervision, J.L. All authors have read and agreed to the published version of the manuscript.

Funding: This research received no external funding.

Institutional Review Board Statement: The study was conducted in accordance with the Declaration of Helsinki, and approved by the Ethics Committee of Central Clinical Hospital of the Ministry of the Internal Affairs and Administration (protocol code 32, 13.02.2019).

Informed Consent Statement: Informed consent was obtained from all subjects involved in the study.

Data Availability Statement: The data presented in this study are available on request from the corresponding authors. The data are not publicly available due to their sensitivity-patient data.

Conflicts of Interest: The authors declare no conflict of interest.

\section{References}

1. Filipov, O. Epidemiology and social burden of the femoral neck fractures. J. IMAB 2014, 20, 516-518. [CrossRef]

2. Gawrońska, K.; Lorkowski, J. Falls, Aging and Public Health-A Literature Review. Ortop. Traumatol. Rehabil. 2020, 22, 397-408. [CrossRef] [PubMed]

3. Gawronska, K.; Lorkowski, J. Falls as One of the Atypical Presentations of COVID-19 in Older Population. Geriatr. Orthop. Surg. Rehabil. 2021, 12, 2151459321996619. [CrossRef] [PubMed]

4. Anandasivam, N.S.; Russo, G.S.; Fischer, J.M.; Samuel, A.M.; Ondeck, N.T.; Swallow, M.S.; Chung, S.H.; Bohl, D.D.; Grauer, J.N. Analysis of Bony and Internal Organ Injuries Associated with 26,357 Adult Femoral Shaft Fractures and Their Impact on Mortality. Orthopedics 2017, 40, e506-e512. [CrossRef] [PubMed]

5. Valentini, R.; Martino, M.; Piovan, G.; De Fabrizio, G.; Fancellu, G. Proximal cut-out in pertrochanteric femural fracture. Acta Biomed. 2014, 85, 144-151.

6. Xu, B.Y.; Yan, S.; Low, L.L.; Vasanwala, F.F.; Low, S.G. Predictors of poor functional outcomes and mortality in patients with hip fracture: A systematic review. BMC Musculoskelet. Disord. 2019, 20, 568. [CrossRef] 
7. Huttunen, T.T.; Kannus, P.; Pihlajamäki, H.; Mattila, V.M. Pertrochanteric fracture of the femur in the Finnish National Hospital Discharge Register: Validity of procedural coding, external cause for injury and diagnosis. BMC Musculoskelet. Disord. 2014, 15, 98. [CrossRef]

8. Deleanu, B.; Prejbeanu, R.; Tsiridis, E.; Vermesan, D.; Crisan, D.; Haragus, H.; Predescu, V.; Birsasteanu, F. Occult fractures of the proximal femur: Imaging diagnosis and management of 82 cases in a regional trauma center. World J. Emerg. Surg. $2015,10,55$. [CrossRef]

9. Meinberg, E.G.; Agel, J.; Roberts, C.S.; Karam, M.D.; Kellam, J.F. Fracture and Dislocation Classification Compendium. J. Ortopeda Trauma 2018, 32, S1-S170. [CrossRef]

10. Young, L.; Uppal, H.S. Hip Fractures: Relevant Anatomy, Classification, and Biomechanics of Fracture and Fixation. Geriatr. Orthop. Surg. Rehabil. 2019, 10. [CrossRef]

11. Saul, D.; Riekenberg, J.; Ammon, J.C.; Hoffmann, D.B.; Sehmisch, S. Hip Fractures: Therapy, Timing, and Complication Spectrum. Orthop. Surg. 2019, 11, 994-1002. [CrossRef] [PubMed]

12. Selim, A.; Ponugoti, N.; Zain Naqvi, A.; Magill, H. Cephalo-medullary nailing versus dynamic hip screw with trochanteric stabilisation plate for the treatment of unstable per-trochanteric hip fractures: A meta-analysis. J. Orthop. Surg. Res. 2021, 16, 47. [CrossRef] [PubMed]

13. Catania, P.; Passaretti, D.; Montemurro, G.; Ripanti, S.; Carbone, S.; Candela, V.; Carnovale, M.; Gumina, S.; Pallotta, F Intramedullary nailing for pertrochanteric fractures of proximal femur: A consecutive series of 323 patients treated with two devices. J. Orthop. Surg. Res. 2019, 14, 449. [CrossRef] [PubMed]

14. Lorkowski, J.; Wilk, R.; Pokorski, M. In Silico Evaluation of Treatment of Periprosthet-ic Fractures in Elderly Patients After Hip Arthroplasty. Adv. Exp. Med. Biol. 2021, 1289, 115-123.

15. Borgström, F.; Karlsson, L.; Ortsäter, G.; Norton, N.; Halbout, P.; Cooper, C.; Lorentzon, M.; McCloskey, E.V.; Harvey, N.C.; Javaid, M.; et al. International Osteoporosis Foundation. Fragility fractures in Europe: Burden, management and opportunities. Arch. Osteoporos. 2020, 15, 59. [CrossRef]

16. Sambrook, P.; Cooper, C. Osteoporosis. Lancet 2006, 367, 2010-2018, Erratum in Lancet 2006, 368, 28. [CrossRef]

17. Khow, K.S.F.; Visvanathan, R. Falls In the Aging Population. Clin. Geriatr. Med. 2017, 33, 357-368. [CrossRef]

18. Pascarella, R.; Fantasia, R.; Maresca, A.; Bettuzzi, C.; Amendola, L.; Violini, S.; Cuoghi, F.; Sangiovanni, P.; Cerbasi, S.; Boriani, S.; et al. How evolution of the nailing system improves results and reduces orthopedic complications: More than 2000 cases of trochanteric fractures treated with the Gamma Nail System. Musculoskelet. Surg. 2016, 100. [CrossRef]

19. Smith, J.R.; Halliday, R.; Aquilina, A.L.; Morrison, R.J.; Yip, G.C.; McArthur, J.; Hull, P.; Gray, A.; Kelly, M.B.; Orthopaedic Trauma Society (OTS). Distal femoral fractures: The need to review the standard of care. Injury 2015, 46, 1084-1088. [CrossRef]

20. Butt, M.S.; Krikler, S.J.; Ali, M.S. Displaced fractures of the distal femur in elderly patients. Operative versus non-operative treatment. J. Bone Jt. Surg. Br. 1996, 78, 110-114. [CrossRef]

21. Hadeed, A.; Werntz, R.L.; Varacallo, M. External Fixation Principles and Overview. In StatPearls; StatPearls Publishing: Treasure Island, FL, USA, 2021.

22. Andrianne, Y.; Hinsenkamp, M. Historical review of the treatment of fractures. Contribution of the Belgian surgery to the origin and development of osteosynthesis. Rev. Med. Brux. 2011, 32, S30-S37. [PubMed]

23. Norris, B.L.; Lang, G.; Russell, T.A.T.; Rothberg, D.L.; Ricci, W.M.; Borrelli, J., Jr. Absolute Versus Relative Fracture Fixation: Impact on Fracture Healing. J. Orthop. Trauma 2018, 1, S12-S16. [CrossRef]

24. Kempf, A.; Grosse, G.; Taglang Favreul, E. Gamma nail in the treatment of closed trochanteric fractures. Results and indications of 121 cases. Orthop. Traumatol. Surg. Res. 2014, 100, 75-83. [CrossRef] [PubMed]

25. Saudan, M.; Lubbeke, A.; Sadowski, C.; Riand, N.; Stern, R.; Hoffmeyer, P. Pertrochanteric fractures; is there an advantage to an intramedullary nail? A randomized, prospective study of 206 patients comparing the dynamic hip screw and proximal femoral nail. J. Orthop. Trauma 2002, 16, 386-393. [CrossRef] [PubMed]

26. Lindskog, D.M.; Braumgaertner, M.R. Unstable intertrochanteric hip fractures in the alderly. J. Am. Acad. Orthop. Surg. 2004, 12, 179-190. [CrossRef]

27. Egol, A.K. Proximal Femur Fractures an Evidence-Based Approach to Evaluation and Management, 1st ed.; Springer: Berlin/Heidelberg, Germany, 2018; pp. 77-100.

28. Lorkowski, J.; Kolaszyńska, O.; Pokorski, M. Artificial Intelligence and Precision Medicine: A Perspective. Adv. Exp. Med. Biol. 2021. [CrossRef]

29. Harris, W.H. Traumatic Arthritis of the Hip after Dislocation and Acetabular Fractures: Treatment by Mold Arthroplasty an end-result study using a new method of results evaluation. J. Bone Jt. Surg. Am. 1969, 51, 737-755. [CrossRef]

30. Babhulkar, S. Unstable trochanteric fractures: Issues and avoiding pitfalls. Injury 2017, 48, 803-818. [CrossRef]

31. Hopp, S.; Wirbel, R.; Ojodu, I.; Pizanis, A.; Pohlemann, T.; Fleischer, J. Does the implant make the difference?-Prospective comparison of two different proximal femur nails. Acta Orthop. Belg. 2016, 82, 319-331.

32. Zhang, H.; Zeng, X.; Zhang, N.; Zeng, D.; Xu, P.; Zhang, L.; Chen, D.; Yu, W.; Zhang, X. INTERTAN nail versus proximal femoral nail antirotation-Asia for intertrochanteric femur fractures in elderly patients with primary osteoporosis. J. Int. Med. Res. 2017, 45, 1297-1309. [CrossRef]

33. Brooks, R. EuroQol: The current state of play. Health Policy 1996, 37, 53-72. [CrossRef] 
34. Griffin, X.L.; Parsons, N.; Achten, J.; Fernandez, M.; Costa, M.L. Recovery of health-related quality of life in a United Kingdom hip fracture population. The Warwick Hip Trauma Evaluation-a prospective cohort study. Bone Jt. J. 2015, 97-B, 372-382. [CrossRef] [PubMed]

35. Parsons, N.; Griffin, X.L.; Achten, J.; Costa, M.L. Outcome assessment after hip fracture: Is EQ-5D the answer? Bone Jt. Res. 2014, 3, 69-75. [CrossRef] [PubMed]

36. Selim, A.A.H.A.; Beder, F.K.; Algeaidy, I.T.; Farhat, A.S.; Diab, N.M.; Barakat, A.S. Management of unstable pertrochanteric fractures, evaluation of forgotten treatment options. SICOT J. 2020, 6, 21. [CrossRef] [PubMed]

37. Mao, W.; Ni, H.; Li, L.; He, Y.; Chen, X.; Tang, H.; Dong, Y. Comparison of Baumgaertner and Chang reduction quality criteria for the assessment of trochanteric fractures. Bone Jt. Res. 2019, 8, 502-550. [CrossRef] [PubMed]

38. Griffin, X.L.; Costa, M.L.; Phelps, E.; Parsons, N.; Dritsaki, M.; Png, M.E.; Achten, J.; Tutton, E.; Lerner, R.; McGibbon, A.; et al. Retrograde intramedullary nail fixation compared with fixed-angle plate fixation for fracture of the distal femur: The TrAFFix feasibility RCT. Health Technol Assess. 2019, 23, 1-132. [CrossRef] [PubMed]

39. Lovejoy, C.O. The origin of man. Science 1981, 211, 341-350. [CrossRef]

40. Coren, S.; Porac, C. Fifty centuries of right-handedness: The historical record. Science 1977, 198, 631-632. [CrossRef]

41. Rogers, L.J.; Vallortigara, G.; Andrew, R. Divided Brains: The Biology and Behaviour of Brain Asymmetries, 1st ed.; Cambridge UP: Cambridge, UK, 2013.

42. Lemon, R.N. Descending pathways in motor control. Annu. Rev. Neurosci. 2008, 31, 195-218. [CrossRef]

43. Versace, E.; Vallortigara, G. Forelimb preferences in human beings and other species: Multiple models for testing hypotheses on lateralization. Front. Psychol. 2015, 6, 233. [CrossRef]

44. Rogers, L.J.; Vallortigara, G. When and Why Did Brains Break Symmetry? Symmetry 2015, 7, 2181-2194. [CrossRef]

45. Vallortigara, G.; Versace, E. Laterality at the Neural, Cognitive, and Behavioral Levels. In APA Handbook of Comparative Psychology: Vol. 1. Basic Concepts, Methods, Neural Substrate, and Behavior; American Psychological Association: Washington, DC, USA, 2017; pp. 557-577.

46. Vallortigara, G.; Rogers, L.J. Survival with an asymmetrical brain: Advantages and disadvantages of cerebral lateralization. Behav. Brain Sci. 2005, 28, 575-588. [CrossRef] [PubMed]

47. Vallortigara, G.; Rogers, L.J.; Bisazza, A. Possible evolutionary origins of cognitive brain lateralization. Brain Res. Rev. 1999, 30, 164-175. [CrossRef]

48. Vallortigara, G. Comparative neuropsychology of the dual brain: A stroll through left and right animals' perceptual worlds. Brain Lang. 2000, 73, 189-219. [CrossRef]

49. Struchtrup, H. Entropy and the Second Law of Thermodynamics-The Nonequilibrium Perspective. Entropy 2020, $22,793$. [CrossRef] [PubMed]

50. Andrew, R.J. (Ed.) Neural and Behavioural Plasticity: The Use of the Domestic Chicken as a Model; Oxford University Press: Oxford, UK, 1991.

51. Vallortigara, G.; Cozzutti, C.; Tommasi, L.; Rogers, L.J. How birds use their eyes: Opposite left-right specialisation for the lateral and frontal visual hemifield in the domestic chick. Curr. Biol. 2001, 11, 29-33. [CrossRef]

52. Jozet-Alves, C.; Viblanc, V.A.; Romagny, S.; Dacher, M.; Healy, S.D.; Dickel, L. Visual lateralization is task and age dependent in cuttlefish, Sepia officinalis. Anim. Behav. 2012, 83, 1313-1318. [CrossRef]

53. Romano, D.; Benelli, G.; Kavallieratos, N.G.; Athanassiou, C.G.; Canale, A.; Stefanini, C. Beetle-robot hybrid interaction: Sex, lateralization and mating experience modulate behavioural responses to robotic cues in the larger grain borer Prostephanus truncatus (Horn). Biol. Cybern. 2020, 114, 473-483. [CrossRef]

54. Ciszkiewicz, A.; Lorkowski, J.; Milewski, G. A novel planning solution for semi-autonomous aspiration of Baker's cysts. Int. J. Med. Robot. 2018, 14. [CrossRef]

55. Frasnelli, E.; Vallortigara, G. Individual-Level and Population-Level Lateralization: Two Sides of the Same Coin. Symmetry 2018, 10, 739. [CrossRef]

56. Romano, D.; Kavallieratos, N.G.; Athanassiou, C.G.; Stefanini, C.; Canale, A.; Benelli, G. Impact of geographical origin and rearing medium on mating success and lateralization in the rice weevil, Sitophilus oryzae (L.) (Coleoptera: Curculionidae). J. Stored Prod. Res. 2016, 69, 106-112. [CrossRef]

57. Guyer, P.; Landolt, M.; Keller, H.; Eberle, C. The Gamma Nail in per- and intertrochanteric femoral fractures-alternative or supplement to the dynamic hip screw? A prospective randomized study of 100 patients with per- and intertrochanteric femoral fractures in the surgical clinic of the City Hospital of Triemli, Zurich, September 1989-June 1990. Aktuelle Traumatol. 1991, 21, 242-249. [PubMed]

58. Gjertsen, J.E.; Baste, V.; Fevang, J.M.; Furnes, O.; Engesæter, B. Quality of life following hip fractures: Results from the Norwegian hip fracture register. BMC Musculoskel. Disord. 2016, 17, 265. [CrossRef] [PubMed]

59. Peeters, C.M.; Visser, E.; Van de Ree, C.L.; Gosens, T.; Den Oudsten, B.L.; De Vries, J. Quality of life after hip fracture in the elderly: A systematic literature review. Injury 2016, 47, 1369-1382. [CrossRef]

60. Cheng, Y.X.; Sheng, X. Optimal surgical methods to treat intertrochanteric fracture: A Bayesian network meta-analysis based on 36 randomized controlled trials. J. Orthop. Surg. Res. 2020, 15, 402. [CrossRef] 
61. Hong, C.C.; Nashi, N.; Makandura, M.C.; Tan, J.H.; Peter, L.; Murphy, D. The long and short of cephalomedullary nails in the treatment of osteoporotic pertrochanteric fracture. Singap. Med. J. 2017, 58, 85-91. [CrossRef]

62. Tsai, S.W.; Lin, C.J.; Tzeng, Y.H.; Lin, C.C.; Huang, C.K.; Chang, M.C.; Chiang, C.C. Risk factors for cut-out failure of Gamma3 nails in treating unstable intertrochanteric fractures: An analysis of 176 patients. J. Chin. Med. Assoc. 2017, 80, 587-594. [CrossRef] 